\title{
Acute viral bronchiolitis in South Africa: Diagnosis and current management
}

This issue of CME profiles acute viral bronchiolitis - a common condition that is often not serious. Nonetheless, each year a number of infants, particularly those with an underlying susceptibility to severe disease, are admitted to hospital or even to paediatric intensive care units. This series of articles highlights the diagnostic principles involved, and emphasises the principles of management and prevention.

Acute viral bronchiolitis occurs primarily in infants $<1$ year of age. It is frequently the first episode of a wheezing illness in a child. This episode should never be labelled asthma, although it may lead to subsequent wheezing and even asthma later in life, which has implications for management and certainly for prevention.

Children develop a common cold and then the viruses involved may produce inflammation in the lower respiratory tract airways. Lower respiratory tract infection, in cases of bronchiolitis, usually has a viral aetiology and does not require antibiotic management. It is distinguished from pneumonia in that, although producing fast breathing, it causes hyperinflation of the chest, which suggests airway involvement - not air-space disease.

Any infant presenting to a doctor with cough and age-specific fast breathing, must be assessed for hypoxia. Peripheral oxygen saturation is the only test routinely required in such children. If hypoxic, the infant must receive oxygen via nasal prongs or face mask and be admitted to hospital.

As bacterial co-infection is rare, testing for bacterial involvement is unnecessary and unreliable in all but the sickest of children. It also implies that therapy should not include the routine use of antibiotics, which is unnecessary and potentially dangerous, as the emergence of resistant pathogens is an important concern with over-use and abuse of these drugs.

Most routine therapies for asthma are ineffective for bronchiolitis and should not be used, including bronchodilators (oral and nebulised), steroids (oral, systemic or nebulised), montelukast and all the over-the-counter cough and cold medicines. A number of studies have shown that physiotherapy, which is so popular in South Africa (SA), worsens hypoxia, and should therefore be discontinued in such cases. While hypertonic saline nebulisation was suggested as an effective therapy for bronchiolitis, reducing the need for and duration of hospitalisation, recent large studies have shown that it is ineffective. It should therefore not be recommended.

There are important preventive modalities for bronchiolitis, including isolation of children with colds (i.e. sick children should not go to crèches for a few days), and teaching children (and adults looking after infants) to practise sneeze and cough hygiene. Prevention does not include the use of prophylactic antibiotics for children with upper respiratory tract infections; their use is strongly discouraged.

Prevention of respiratory syncytial virus (RSV) disease in young children may be the most effective strategy to reduce the burden of disease, especially in children at high risk of developing severe disease. ${ }^{[1]}$ The monoclonal antibody, palivizumab, administered monthly throughout the RSV season to infants and children at high risk of severe RSV disease, has been shown to be effective for prevention. Furthermore, palivizumab has also been proven to reduce RSV disease, wheezing and recurrent wheezing episodes in the first year of life when administered to late preterm infants. ${ }^{\left[{ }^{[2]}\right.}$
However, its use is limited by its high cost and the need for monthly intramuscular injections during the RSV season. More recently, increased attention has been given to the development of an effective RSV vaccine, particularly targeted at pregnant women, as a strategy for prevention of RSV disease in their offspring in the first months of life. ${ }^{[3]}$ This is an attractive strategy for which the results of clinical trials are awaited.

The two CME articles in this edition of SAMJ are a concise reflection of the evidence with regard to acute viral bronchiolitis. The guideline committee has reviewed the available literature in making these recommendations. Each section has been fully referenced. The first $\operatorname{article}^{[4]}$ gives a step-wise approach to the diagnosis of acute viral bronchiolitis, suggesting that 'less is more' by way of tests in patients with this condition; that many tests are inappropriate; and that important tests such as peripheral oxygen saturation must be appropriately performed. The second article ${ }^{[5]}$ is a summary of what should be used with regard to treatment and prevention.

A number of guidelines for the management and prevention of acute viral bronchiolitis are published worldwide annually. These have been consulted in our recommendations. However, none perfectly fits the disease entity, organism seasonality, duration and specific considerations for SA. Hence the need for local guidelines on this topic. It is hoped that these will guide local healthcare practitioners, funders and policy makers in ensuring the best care of children in SA.

\section{R J Green}

Guest editor

Department of Paediatrics and Child Health, Faculty of Health Sciences, University of Pretoria, and Steve Biko Academic Hospital, Pretoria, South Africa robin.green@up.ac.za

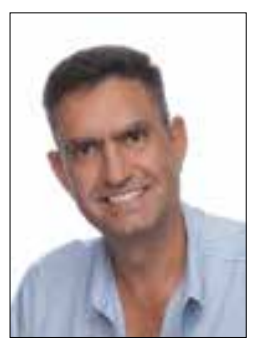

\section{H J Zar}

Guest editor

Department of Paediatrics and Child Health, Red Cross War Memorial Children's Hospital, and MRC Unit on Child and Adolescent Health, Faculty of Health Sciences,

University of Cape Town, South Africa heather.zar@uct.ac.za

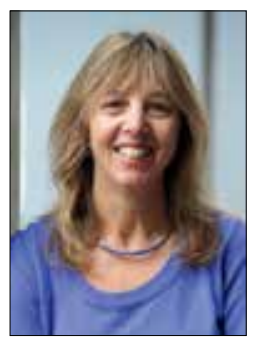

1. Shi T, Balsells E, Wastnedge E, et al. Risk factors for respiratory syncytial virus associated with acute ower respiratory infection in children under five years: Systematic review and meta-analysis. J Glob Health 2015;5(2):020416. DOI:10.7189/jogh.05.020416

2. Blanken MO, Rovers MM, Molenaar JM, et al. Respiratory syncytial virus and recurrent wheeze in healthy preterm infants. N Engl J Med 2013;368(19):1791-1799. DOI:10.1056/NEJMoa1211917

. Mazur NI, Martinón-Torres F, Baraldi E, et al. Lower respiratory tract infection caused by respiratory syncytial virus: Current management and new therapeutics. Lancet Respir Med 2015;3(11):888-900. DOI:10.1016/S2213-2600(15)00255-6

White DA, Zar HJ, Madhi SA, et al. Acute viral bronchiolitis in South Africa: Diagnostic flow. S Afr Med J 2016;106(4):328-329. DOI:10.7196/SAMJ.2016.v106i4.10441

Zar HJ, Madhi SA, White DA, et al Acute viral bronchiolitis in South Africa: Strategies for management and prevention. S Afr Med J 2016;106(4):330-332. DOI:10.7196/SAMJ.2016.v106i4.10437 